\title{
Does reduction of the oncologic safety margin for facial basal cell carcinoma result in higher recurrence rates?
}

\author{
Eon Su Kim, \\ Chae Eun Yang, \\ Yoon Kyu Chung \\ Department of Plastic and \\ Reconstructive Surgery, Wonju \\ Severance Christian Hospital, Yonsei \\ University Wonju College of Medicine, \\ Wonju, Korea
}

\begin{abstract}
Background: Wide surgical excision is the gold standard for basal cell carcinoma (BCC) treatment. Typically, resection requires a safety margin $\geq 4 \mathrm{~mm}$. We aimed to confirm BCC excisions' cancer recurrence rate and safety on the facial region with new safety margins.

Methods: We included patients with primary BCC on the facial region who underwent wide excision with 2- or 3-mm safety margins at our institution between January 2010 and December 2018. Medical records were reviewed to confirm the epidemiology and surgical information. Recurrence was confirmed by physical examination through regular 6-month follow-up.

Results: We included 184 out of 233 patients in this study after applying the exclusion criteria. The mean age and follow-up period were $71.2 \pm 10.2$ years and $29.3 \pm 13.5$ months, respectively. The predominantly affected area was the nose ( 95 cases); a V-Y advancement flap was the most commonly used surgical method. There were two cases of recurrence in the $2 \mathrm{~mm}$ margin group and one recurrence in the group resected with $3 \mathrm{~mm}$ margins.

Conclusion: In this large cohort study, we found 2-3 mm excision margins can yield enough safety in facial BCCs. The recurrence rates were found to be comparable with those reported after wider margins.
\end{abstract}

Abbreviations: BCC, basal cell carcinoma; FTSG, full-thickness skin graft; STSG, split-thickness skin graft.

Keywords: Basal cell carcinoma / Local neoplasm recurrence / Resection margin / Skin neoplasms

\section{INTRODUCTION}

Basal cell carcinoma (BCC) is the most common type of nonmelanoma skin cancer [1-3]. According to an American Cancer Society estimate, 5.4 million patients were diagnosed with non-melanoma cancer in 2012, and $80 \%$ of these were BCC [46]. Nearly 2.8 million new BCC cases are identified each year in

\footnotetext{
Correspondence: Chae Eun Yang

Department of Plastic and Reconstructive Surgery, Wonju Severance Christian

Hospital, Yonsei University Wonju College of Medicine, 20 Ilsan-ro, Wonju 26426,

Korea

E-mail: pscey@yonsei.ac.kr

Received May 20, 2021 / Revised June 4, 2021 / Accepted June 10, 202
}

the United States alone. BCC is a slow-growing skin tumor, and fatal metastasis to other organs rarely occurs [7]. However, some BCCs infiltrate tissues in an irregular three-dimensional fashion through finger-like projections [8]. If left untreated, BCC destroys and invades adjacent tissues. Therefore, complete surgical resection is considered the gold standard for treatment $[9,10]$.

For skin cancer surgeries, the most important aspect of complete surgical resection is the determination of the resection margin that allows complete tumor resection while minimizing damage to the normal tissue. There are various opinions on the appropriate safety margins. The previous trend stated that a 
4-mm safety margin was the most effective [9,11-14]. Although the difference between $2 \mathrm{~mm}$ and $4 \mathrm{~mm}$ does not seem large, in small areas such as the medial canthus, eyelid, and ala, even a few millimeters can lead to noticeable structural changes in the facial region. In addition, depending on the BCC subtype, in types other than nodular and superficial BCC, incomplete resections can occur commonly [11].

Approximately $80 \%$ of BCCs occur in the head and neck. Particularly, since resections as small as $1 \mathrm{~mm}$ on the face can lead to unfavorable structural changes, we reduced the resection margins to $2 \mathrm{~mm}$ and $3 \mathrm{~mm}$ to study oncological safety after resection [15].

Even small changes in the face cause structural changes, and we studied whether the safety margin of $4 \mathrm{~mm}$ can be reduced. Therefore, we performed surgery by reducing the resection margin to $2 \mathrm{~mm}$ or $3 \mathrm{~mm}$ for patients treated with BCC in our institution from January 2010 to December 2018. We conducted a study to confirm the recurrence rate and safety of this method.

\section{METHODS}

\section{Study design and setting}

We conducted a retrospective study of 233 patients with a primary BCC diagnosis who underwent wide excision at our hospital from January 2010 to December 2018.

\section{Patient selection and medical record review}

We reviewed 233 patients who underwent surgical resection after being diagnosed with BCC in our institution during this study. Of these, 49 patients who missed outpatient follow-up, had radiation therapy, relapsed, had immunosuppressive disorders, or were on immunosuppressive medication were excluded, and data were analyzed for the remaining 184 patients. The patients' medical records were reviewed to collect data on their age, location, surgery method, safety margin, additional frozen section diagnosis, clinical appearance, and BCC recurrence. All patient information was anonymized. This study was approved by the Institutional Review Board of Wonju Severance Christian Hospital (IRB No. CR 320046).

\section{Variables}

We investigated variables that could affect recurrence, i.e., age, location, surgical method, safety margin, additional frozen section diagnosis, and gross appearance. Evaluation of the results was compared and analyzed through the recurrence rate. To eliminate surgeon bias, the study included patients operated on by a single surgeon.

\section{Measurement}

\section{Surgical methods}

Photographs were taken before and after the operation. To determine the safety margin, we observed the gross appearance to establish the extent of resection while considering the following specifics. For lesions that were well-defined, pink or flesh-colored papules or nodules, well-defined ulcers, rolled borders, or presence of erythematous macules with crust or scale, we performed excision with a 2-mm margin. Lesions that were white with ill-defined borders, ulcerative, with slightly elevated or depressed areas of induration and telangiectasia, were resected with a 3-mm surgical margin (Fig. 1). All lesions were excised at the subcutaneous level.

To immediately identify residual cancer cells after tumor resection, a frozen section test was performed at the base of the lesion and clock angles of 3, 6, 9, and 12 oclock to confirm that there were no residual cancer cells. Surgical blade no. 11 was used to collect 1-mm-sized tissue sections from the aforementioned locations. When residual cancer cells were identified in the frozen section test, additional resection was performed until residual cancer cells were no longer visible. The excised specimen was fixed in formalin and sent to the pathology laboratory, where it was embedded in paraffin, processed, and stained for tissue margin evaluation. Reconstruction techniques such as primary repair, local and V-Y advancement flap, and skin graft were used to cover the skin defects.

\section{Recurrence evaluation}

Follow-up was performed at 6-month intervals to rule out recurrence. When there were suspicious lesions or clinical symptoms such as color change, itching, redness, and wounds without a specific etiology, a biopsy was performed to confirm recurrence. Recurrence was diagnosed when BCC was confirmed on pathological examination.

\section{Statistical methods}

Data were analyzed using IBM SPSS Statistics for Windows, version 22.0 (IBM Corp., Armonk, NY, USA). The recurrence rate of BCC was calculated following wide excision. Univariate logistic regression was used to evaluate variables associated with recurrence. All tests were two-sided, and $p<0.05$ was considered statistically significant.

\section{RESULTS}

In total, we reviewed the medical records of 184 patients. The average age and follow-up period were $71.2 \pm 10.2$ years (range, 41-95 years) and $29.3 \pm 13.5$ months (range, 6-116 months), 

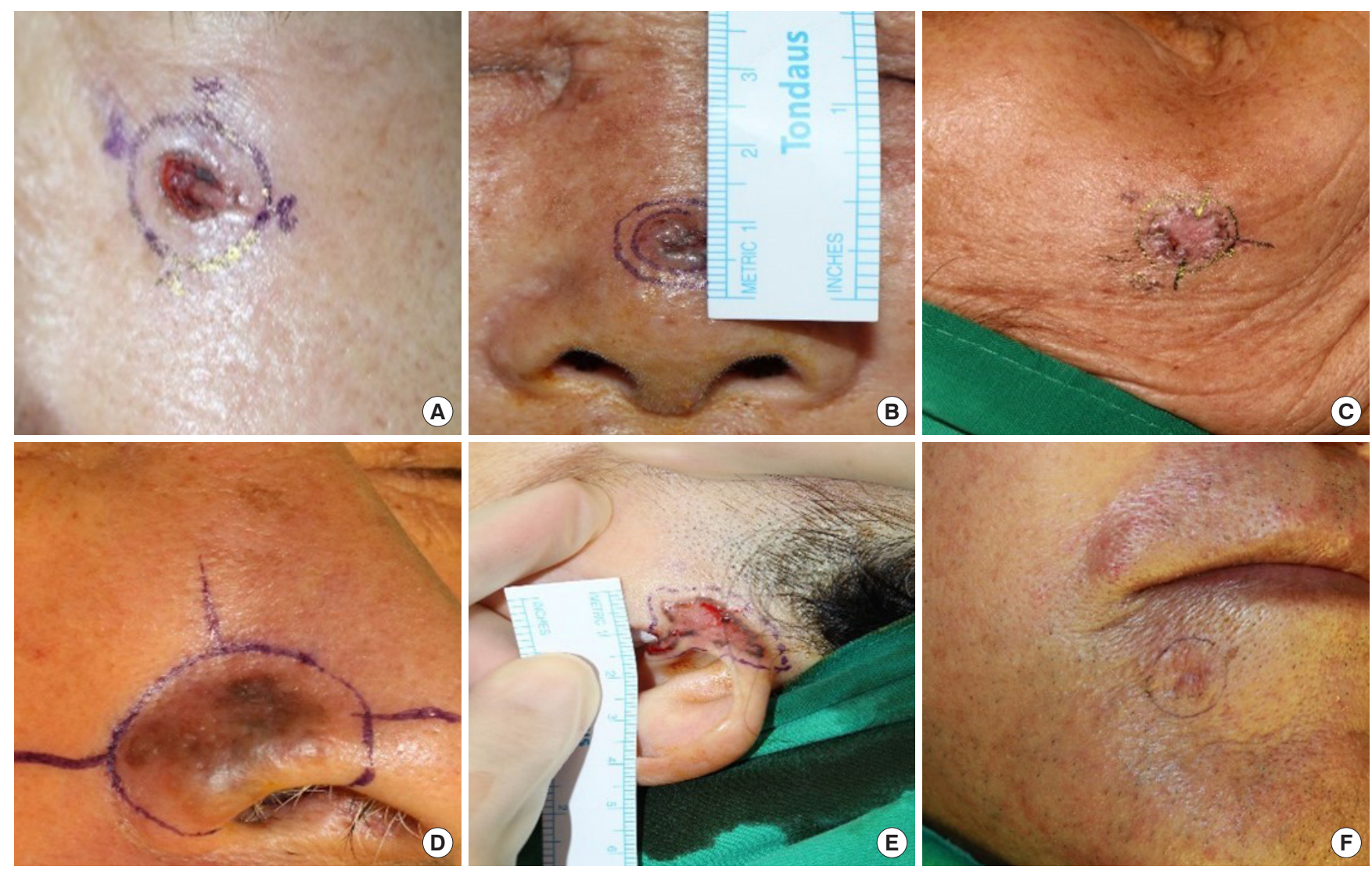

Fig. 1. Margin demarcation according to the gross appearance. Wide excision was performed with a safety margin of $2 \mathrm{~mm}$ (A-C) or $3 \mathrm{~mm}$ (D-F) depending on the gross appearance of basal cell carcinoma in various areas.

respectively. We used a surgical margin of $2 \mathrm{~mm}$ and $3 \mathrm{~mm}$ in 134 and 50 patients, respectively.

Of the 184 patients, 95 had surgeries performed on their noses, and 10 had surgeries performed on the head and neck. Other surgical sites included the forehead, chin, and ear. Also, a $\mathrm{V}-\mathrm{Y}$ advancement flap, which was used in 71 cases, was the most common method used to cover the defect. Limberg flaps, skin grafts, and primary repairs were also included (Table 1).

Reconstruction was performed if no cancer cells were found during a simultaneous frozen section examination. In four cases, cancer cells were identified in the frozen section examination, and additional resection was required. Two cases in both the 2-mm and 3-mm margin groups had positive margins in the frozen section test. In those cases, an additional $1 \mathrm{~mm}$ area was resected in the positive direction, and reconstruction was performed after confirming a negative frozen section test. No cancer cells were found in the margins of the permanent specimens of these patients. We confirmed three cases of recurrence (two with 2-mm and one with 3-mm safety margin, respectively) that required additional surgery during the follow-up period. In all three cases, cancer cells were not observed in either the frozen section examination or the permanent specimen at the time of surgery.

No significant interactions were observed between variables that were eligible for the multivariate model. The following variables were not statistically associated with recurrence: sex, operation type, site, and additional frozen section examination $(p>0.05)$.

Patients who experienced recurrence were reviewed at 35,43 , and 44 months. In patients with 2-mm resection, we observed well-defined ulcerative lesions or erythematous macules. On the other hand, in patients with 3-mm resections, white, depressed lesions with ill-defined borders were observed (Table 2). The recurrence rates were $1.5 \%$ and $2 \%$ in the patients with 2-mm and 3-mm resection margins, respectively (Fig. 2). In the cases of recurrence, wide excision was performed with a resection margin of $3 \mathrm{~mm}$. Cancer cells were not found in the permanent specimen or in the frozen section.

\section{DISCUSSION}

In the surgical treatment of BCC, the range of surgical margin has been well researched since Wolf and Zitelli [11] proposed a 4-mm surgical margin in 1987. However, it is difficult to deter- 
mine the extent of cancer removal as it mainly appears on the face of elderly patients [15]. The recurrence rate after BCC surgery varies from $3 \%$ to $16 \%$, and it is generally accepted that a safety margin of $4 \mathrm{~mm}$ or more is appropriate $[2,16,17]$.

Table 1. Patient epidemiology and management

\begin{tabular}{lc}
\hline Characteristic & Value $(n=184)$ \\
\hline Sex, male/female & $70 / 114$ \\
Age (yr) & $71.2 \pm 10.2$ \\
Follow-up period (mo) & $29.3 \pm 13.5$ \\
Surgical margins & \\
2-mm margin & 134 \\
3-mm margin & 50 \\
Site & \\
Cheek & 24 \\
Chin & 1 \\
Ear & 7 \\
Forehead & 10 \\
Lip & 8 \\
Nose & 95 \\
Periorbital & 16 \\
Scalp & 9 \\
Temple & 4 \\
Others & 10 \\
Type of operation & 17 \\
V-Y advancement flap & 4 \\
Limberg flap & 47 \\
STSG & 47 \\
Primary repair & \\
FTSG & \\
Composite graft & \\
\hline & \\
\hline
\end{tabular}

Values are presented as number or mean $\pm S D$. In the case of patient sex and age, follow-up period, surgical margin, site, type of operation, the data are calculated from the patients studied.

STSG, split-thickness skin graft; FTSG, full-thickness skin graft.
We hypothesized that smaller resection margins will not lead to a high recurrence rate and conducted research with new 2and 3-mm margins in the facial region (Fig. 1). Unlike previous studies, we did not determine the resection margin according to the size, histological type, or location of the lesion, but through clinical appearance $[11,18]$. In this study, we performed wide excision with a $2-\mathrm{mm}$ or 3-mm surgical margin using the gross appearance presented in the "Methods" section.

While recurrence was confirmed in three cases, there were no cancer cells on the frozen section examinations or permanent specimens of these patients. We found that our recurrence rates were comparable with those of previous studies [2,16,17]. All lesions were covered with a skin graft or local flap. In previous studies, resection was deemed necessary if the frozen section test was abnormal [19]. However, in this study, when atypical dysplasia other than cancer cells was found on the frozen section test, no additional resection was performed. Ultimately, we learned that there was no evidence for recurrence without further resection. Other variables, such as sex, operation type, site, and additional frozen section examination, did not have a sta-

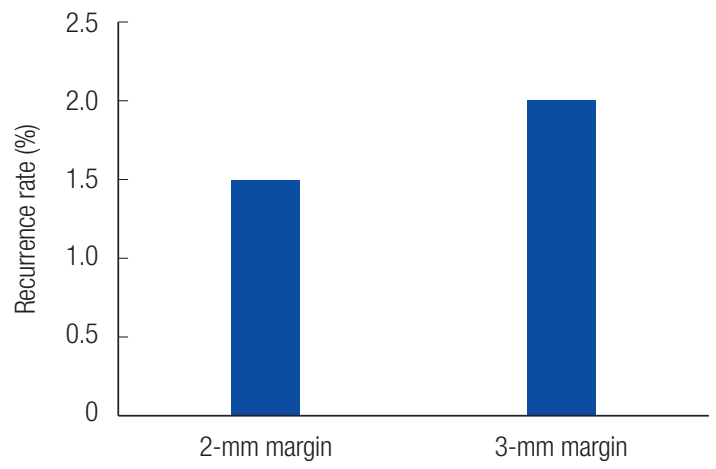

Fig. 2. The recurrence rate of each safety margin. We found recurrence in two cases with 2-mm safety margin and one case with 3-mm safety margin. Recurrence rates of $1.5 \%$ and $2 \%$ were confirmed for each safety margin.

Table 2. Details of patients with basal cell carcinoma recurrence

\begin{tabular}{lccc}
\hline Patient details & \multicolumn{2}{c}{ Patient No. } & 3 \\
\cline { 2 - 4 } & 1 & 2 & Male \\
Sex & Male & 64 & 73 \\
Age (yr) & 85 & $0.5 \times 0.5$ & $0.8 \times 0.8$ \\
Primary cancer size (cm) & $0.8 \times 0.7$ & Nose & Nose \\
Site & Nose & Erythematous macule with crust lesion & White-colored, depressed lesions with ill-defined borders \\
Gross appearance & Well-defined ulcerative lesion & 2 & 3 \\
Safety margin (mm) & 2 & V-Y advancement flap & STSG \\
Reconstruction & V-Y advancement flap & 43 & 44 \\
Recurrence (mo) & 35 & & \\
\hline
\end{tabular}

Recurrence was confirmed in three patients. Two cases recurred when resected with a safety margin of $2 \mathrm{~mm}$, and one case recurred when resected with a safety margin of 3 $\mathrm{mm}$.

STSG, split-thickness skin graft. 
tistically significant relationship with recurrence.

The recurrence rate varies depending on cancer's histologic type; however, it cannot be confirmed during surgery. As a result, the operator has no choice but to determine the extent of the resection by judging the gross appearance. This study is meaningful in that it is a large cohort study that can confirm that the recurrence rate does not increase when a small area is resected with gross appearance.

In this study, the frozen section examination was not performed continuously as in Mohs surgery, we performed the frozen section test on several parts of the lesion, which was simpler and had a lower recurrence rate than the method used in Mohs surgery. In previous studies, Mohs surgery has had no significant advantage in primary BCC treatment, and it required a significant amount of time and resources from the patient and additional training for the surgeon $[17,20]$.

There were a few limitations to this study. First, since the histological subtype and size were not investigated, we did not conduct studies on the related variables. Second, the study size was limited to 184 cases, making continuous follow-up difficult for patients due to factors such as low compliance and death due to old age. Third, this also made statistical analysis difficult. Therefore, we propose that a study on the recurrence rate through continuous follow-up be conducted. Fourth, this was a retrospective study, and we did not include a control group. Fifth, bias may exist, as this was a single-institution study of cases operated on by a single surgeon. Sixth, since there was no evaluation of the cosmetic results, further research may be needed to investigate patient satisfaction with the aesthetic aspects of the procedure.

In this study, we aimed to study the cancer recurrence rate and safety of BCC excisions with 2- or 3-mm safety margins. We conclude that performing 2- or 3-mm resection in the facial region according to the clinical appearance is comparable with previously proposed methods as the procedures reduced the defect size with no difference in the recurrence rate.

\section{NOTES}

\section{Conflict of interest}

No potential conflict of interest relevant to this article was reported.

\section{Ethical approval}

The study was approved by the Institutional Review Board of Wonju Severance Christian Hospital (IRB No. CR 320046) and performed in accordance with the principles of the Declaration of Helsinki.

\section{Patient consent}

The patients provided written informed consent for the publication and the use of their images.

\section{ORCID}

Eon $\mathrm{Su} \mathrm{Kim}$

https://orcid.org/0000-0001-5938-9645

Chae Eun Yang

https://orcid.org/0000-0001-8128-797X

Yoon Kyu Chung

https://orcid.org/0000-0002-0401-3912

Author contribution

Conceptualization: CEY, YKC. Data curation: ESK. Visualization: ESK, CEY, YKC. Writing - original draft: ESK. Writing review \& editing: ESK, CEY.

\section{REFERENCES}

1. Chren MM, Linos E, Torres JS, Stuart SE, Parvataneni R, Boscardin WJ. Tumor recurrence 5 years after treatment of cutaneous basal cell carcinoma and squamous cell carcinoma. J Invest Dermatol 2013;133:1188-96.

2. Mendez BM, Thornton JF. Current basal and squamous cell skin cancer management. Plast Reconstr Surg 2018;142:373e387 e.

3. Mohan SV, Chang AL. Advanced basal cell carcinoma: epidemiology and therapeutic innovations. Curr Dermatol Rep 2014;3:40-5.

4. Dacosta Byfield S, Chen D, Yim YM, Reyes C. Age distribution of patients with advanced non-melanoma skin cancer in the United States. Arch Dermatol Res 2013;305:845-50.

5. American Cancer Society. Cancer Facts \& Figures 2020 [Internet]. Atlanta, GA: American Cancer Society; c2020 [cited 2021 Jan 16]. Available from: https://www.cancer.org/research/cancer-facts-statistics/all-cancer-facts-figures/cancer-facts-figures-2020.html.

6. Park YJ, Kwon GH, Kim JO, Kim NK, Ryu WS, Lee KS. A retrospective study of changes in skin cancer characteristics over 11 years. Arch Craniofac Surg 2020;21:87-91.

7. Walling HW, Fosko SW, Geraminejad PA, Whitaker DC, Arpey CJ. Aggressive basal cell carcinoma: presentation, pathogenesis, and management. Cancer Metastasis Rev 2004;23:389402.

8. Breuninger H, Dietz K. Prediction of subclinical tumor infiltration in basal cell carcinoma. J Dermatol Surg Oncol 1991; 17:574-8.

9. Gulleth Y, Goldberg N, Silverman RP, Gastman BR. What is the best surgical margin for a Basal cell carcinoma: a metaanalysis of the literature. Plast Reconstr Surg 2010;126:1222-31. 10. Marzuka AG, Book SE. Basal cell carcinoma: pathogenesis, ep- 
idemiology, clinical features, diagnosis, histopathology, and management. Yale J Biol Med 2015;88:167-79.

11. Wolf DJ, Zitelli JA. Surgical margins for basal cell carcinoma. Arch Dermatol 1987;123:340-4.

12. Bichakjian CK, Olencki T, Aasi SZ, Alam M, Andersen JS, Berg D, et al. Basal cell skin cancer, version 1. 2016, NCCN Clinical Practice Guidelines in Oncology. J Natl Compr Canc Netw 2016;14:574-97.

13. Thomas DJ, King AR, Peat BG. Excision margins for nonmelanotic skin cancer. Plast Reconstr Surg 2003;112:57-63.

14. Wee SJ, Park MC, Chung CM. Basal cell carcinoma misdiagnosed as trichoepithelioma. Arch Craniofac Surg 2020;21:2025.

15. Wong CS, Strange RC, Lear JT. Basal cell carcinoma. BMJ 2003;327:794-8.

16. Cameron MC, Lee E, Hibler BP, Giordano CN, Barker CA,
Mori S, et al. Basal cell carcinoma: contemporary approaches to diagnosis, treatment, and prevention. J Am Acad Dermatol 2019;80:321-39.

17. Shelton ME, Adamson AS. Review and update on evidencebased surgical treatment recommendations for nonmelanoma skin cancer. Dermatol Clin 2019;37:425-33.

18. Walker P, Hill D. Surgical treatment of basal cell carcinomas using standard postoperative histological assessment. Australas J Dermatol 2006;47:1-12.

19. Nicoletti G, Brenta F, Malovini A, Musumarra G, Scevola S, Faga A. Study to determine whether intraoperative frozen section biopsy improves surgical treatment of non-melanoma skin cancer. Mol Clin Oncol 2013;1:390-4.

20. Cumberland L, Dana A, Liegeois N. Mohs micrographic surgery for the management of nonmelanoma skin cancers. Facial Plast Surg Clin North Am 2009;17:325-35. 